\title{
Des densités aux rugosités. Les modalités instrumentales d'une
} transposition

Jean-Pierre Peneau

\section{Citer ce document / Cite this document :}

Peneau Jean-Pierre. Des densités aux rugosités. Les modalités instrumentales d'une transposition . In: Les Annales de la recherche urbaine, $\mathrm{N}^{\circ} 67,1995$. Densités et espacements. pp. 128-134;

doi : https://doi.org/10.3406/aru.1995.1884

https://www.persee.fr/doc/aru_0180-930x_1995_num_67_1_1884

Fichier pdf généré le 23/04/2018 


\title{
Zusammenfassung
}

Von der «Dichtigkeit» zur «Rauhigkeit» : Modalitäten einer Transposition

Luftströmungen und die mit innen einhergehenden Umweltbelastungen hängen mit Bebauungsdichte und Bauvolumen zusammen. Interdisziplinäre Studien versuchen, anhand eines kartographischen Prototyps der «Rauhigkeit» von Städten ihre Effekte zu simulieren und eine Maßeinheit zu bestimmen. In Zukunft dürften die in Erfahrung zu gebrachten Indikatoren auch für die Untersuchung von Lichtwirkungen, Lärmimmission und thermischem Klima aufschlußreich sein.

\section{Résumé}

La densité en surface et en volume des espaces urbains joue sur les déplacements d'air et leurs nuisances. Plusieurs disciplines se sont associées pour en définir la mesure et en simuler les effets dans un prototype cartographique de rugosité urbaine. Au-delà de cette première expérience, les indicateurs mis à jour pourraient servir à terme de base à des informations sur les ambiances lumineuses, la propagation du bruit ou le climat thermique.

\begin{abstract}
From density to rugosity : the instrumental modalities of a transposition The surface and volume density of urban spaces influences the circulation of air and its noxious effects. Several disciplines worked together to measure it and simulate its effects in a cartographic prototype of urban rugosity. Beyond this preliminary experiment, the parameters brought to light could one day serve as a basis for measuring light levels, noise propagation and thermal climates.
\end{abstract}

\section{Resumen}

De las densidades a las rugosidades : las modalidades instrumentales de una transposición La densidad superficial y volumétrica de los espacios urbanos influye en los desplazamientos de aire y las molestias provocadas por éstos. Varias disciplinas se han asociado para definir la manera de medirlos y simular sus efectos en un prototipo cartográfico de rugosidad urbana. Más allá de esta primera experiencia, los indicadores revelados podrian a la larga servir de base a informaciones sobre los ambientes luminosos, la propagación del ruido o el clima térmico. 


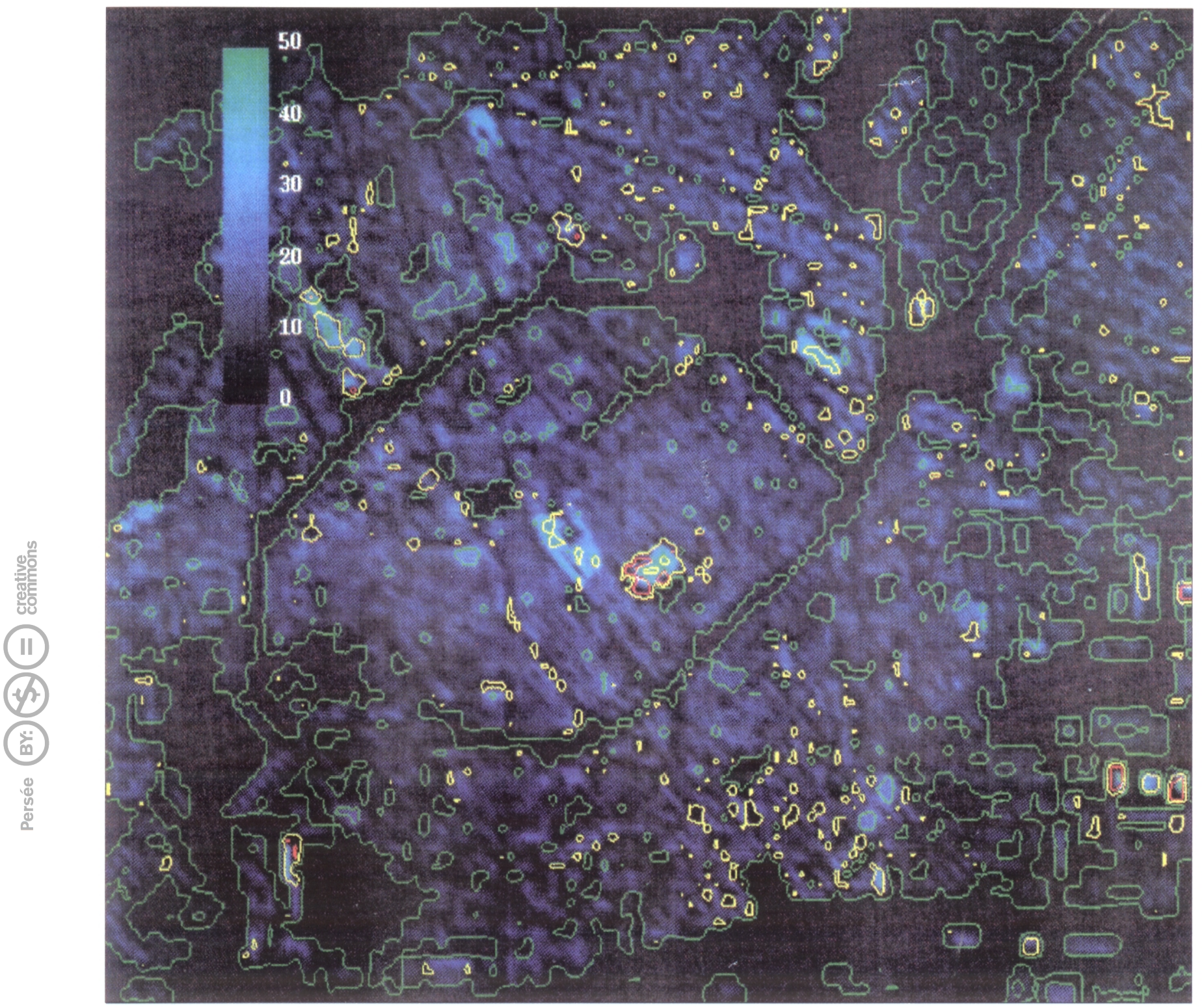

Figure 2:

Deux indicateurs de rugosité pour la ville de Strasbourg sur une maille de 20 mètres par 20 mètres obtenus à partir d'un fichier TRAPU. La couleur bleue correspond à la hauteur surfacique etles lignes de niveau àl'écart-type.

Jean-Pierre Peneau, architecte et urhaniste de formation, est professeur à l' Ecole d'Architecture de Nantes. Il a créé et dirige au sein de cet établissement le Laboratoire CERMa qui a été agréé par le Ministère de l' Equipement puis associé au CNRS. Il est co-responsable du DEA «Ambiances Architecturales et Urbaines» et a été chargé par le PIR-Villes de créer et de coordonner le pôle thématique : "Physique et Images de la Ville». Ce dernier regroupe actuellement douze équipes de Nantes et du Mans.

Ses recherches portent sur les domaines de la simulation des phénomènes physiques et climatiques en milieu urbain et sur les modes d'intégration des techniques correspondantes dans la mise en forme du projet. En regard de cette thématique privilégiée au CtRMA, il mène parallèlement un trar'ail sur les formes anciennes de l'ajustement climatique des dispositions urbaines. 


\title{
DES DENSITÉS AUX RUGOSITÉS
}

\author{
LES MODALITÉS INSTRUMENTALES D'UNE TRANSPOSITION
}

Jean-Pierre Peneau

E dépit des frayeurs millénaristes sur l'effet de serre et sur le réchauffement, force est de constater que le vent mauvais qui emportait le poète souffle toujours sur nos villes. On pourrait même estimer que depuis le XIXe siècle son côté maléfique s'est aggravé : il s'est chargé de substances plus nocives, il propage des bruits et des odeurs plus gênantes, il accélère une course affolée par des obstacles, dont la taille et les distributions n'ont plus rien à voir avec celles des édifices du Paris d'Haussmann ou de Rambuteau.

Ainsi, la seule question de l'écoulement plus ou moins rapide et turbulent du vent dans la ville - qui pourrait être vite cataloguée dans le registre des effets secondaires en matière d'aménagement urbain - retrouve un certain niveau d'acuité, dans sa composition avec d'autres facteurs physiques et climatiques et d'autres phénomènes générateurs de nuisances sévères. Il est clair que de meilleures connaissances en aérodynamique urbaine permettront des avancées dans les prédictions respectives de la diffusion des polluants, de l'amplification des phénomènes sonores, des déperditions énergétiques, du confort piétonnier des espaces extérieurs.

En regard de la double complexité de la physique des écoulements turbulents et de la morphologie du cadre urbain, les techniques de simulation numérique représentent une voie de recherche et de développement particulièrement stimulante et prometteuse. Elles constituent un domaine et un outillage communs pour les trois équipes qui ont été associées à l'occasion de ce projet et de l'appel d'offre du PIR-Villes sur les densités. Mais ce partage, s'il gageait, a priori, la possibilité de la collaboration, n'aurait pas été suffisant pour mettre en œuvre l'action de recherche que l'on se propose d'exposer. Qu'il soit cependant permis de faire précéder sa présentation de quelques éléments très généraux, permettant d'en évoquer le cadre scientifique.

\section{Bref état de l'art en climatologie urbaine}

La climatologie, la micro-climatologie et, a fortiori, la nano-climatologie urbaines, représentent des domaines complexes pour lesquels on ne dispose pas d'un corpus de connaissances bien structuré. Les savoirs sur le sujet résultent des retombées de recherche dans des secteurs connexes : météorologie, étude de dispersion de polluants, ingénierie aérodynamique. Dans ce dernier secteur, en particulier, on ne compte plus les études sur le champ de vitesse ou de pression intéressant des constructions isolées, mais la description du flux et de la dispersion dans l'ensemble d'un quartier, plus modestement dans un fragment urbain, s'avèrent extrêmement rares. Les travaux de l'équipe de T.R. Oke à Vancouver viennent brillamment témoigner dans cette rareté.

Les connaissances actuelles en climatologie urbaine proviennent de trois sources : la simulation en soufflerie ou

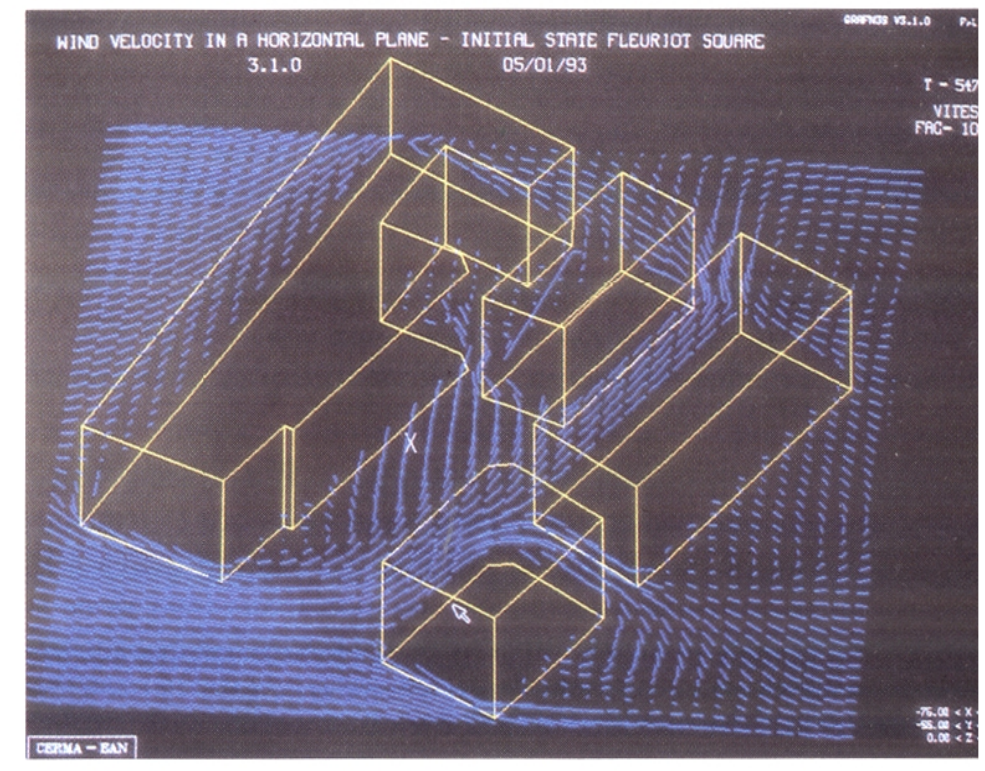

Fig. 1 : Simulation de l'écoulement aérodynamique pour un secteur du centre de Nantes utilisantle code N3S.

en tunnel hydraulique, les mesures sur site, enfin les simulations numériques. Les plus anciennes ont concerné les perturbations liées aux grandes villes à l'échelle de la méso-climatologie. Elles donnent peu d'informations en matière de micro-climatologie urbaine. Plus récemment le développement des puissances des calculateurs a permis la mise en œuvre de

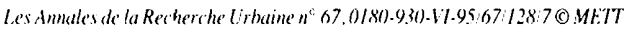


codes de calcul repérés par l'acronyme CFD' (pour Computational Fluid Dynamics). Ils autorisent la prédiction des champs de vitesse et de pression autour d'un bâtiment, puis, en vertu de progrès très récents, dans un ensemble de constructions plus complexe ${ }^{2}$. Un exemple de ce genre de simulation est donné fig. 1.

Il faudrait aussi noter qu'à ces données, viennent dorénavant s'ajouter des informations provenant des observations par satellite.

En matière de climatologie urbaine, le phénomène le plus étudié et le plus notoire est celui de l'îlot de chaleur. Il peut être facilement perçu par la météorologie empirique du quotidien et a fortiori mesuré précisément, mais il est beaucoup moins aisé d'évaluer les effets de la concentration urbaine en matière des flux de chaleur sensible et d'humidité, en termes de dispersion des polluants et des aérosols, ou d'altération du régime d'écoulement des vents.

Le problème principal est celui de la structure des flux correspondants à l'intérieur de cette zone délimitée par le toit virtuel continu de la villc, dćsignć par l'cxpression dc canopée urbaine. Celle-ci sera liée à la hauteur et à la densité des constructions et des obstacles naturels qui jalonnent l'espace urbain.

Au sein de la couche séparant le sol de la canopée urbaine, les recherches portent sur le bilan énergétique global, sur la dispersion des polluants, et sur le confort des espaces extérieurs. Les interactions entre ces niveaux d'investigation sont nombreuses et les voies de la simulation numérique paraissent tout indiquées, pour explorer les effets croisés des divers paramètres physiques et climatiques concernés 3 .

\section{Un projet de recherche interdisciplinaire}

Le projet de recherche retenu par le PIR-Villes s'inscrit dans une action concertée des spécialistes de l'aérodynamique et de la climatologie qui sont attachés à la construction d'un modèle communautaire français de la couche limite atmosphérique. Ce modèle est désigné par les préfixes «sub-méso» qui dans le langage des scientifiques concernés correspond à des échelles situées entre 30 mètres et 30 kilomètres. L'objectif à terme de cette action est de permettre la simulation numérique détaillée :

- de la dynamique des écoulements de l'air ;

- de la diffusion turbulente des polluants ;

- de la microphysique et de la chimie troposphérique audessus des agglomérations urbaines.

Les partenaires de l'opération, au nombre d'une douzaine, représentent une gamme d'organismes et d'institutions allant du constructeur aéronautique au laboratoire universitaire. Ils se sont constitués en groupement de recherche sous l'égide du CNRS et sous l'appellation qui pourrait paraitre elliptique de «GDR sub-méso» ${ }^{4}$.
C'est au sein de ce Groupement de Recherche que la question du paramétrage des conditions aux limites des simulations numériques s'est posée. En effet, aux échelles considérées il est indispensable de remplacer le calcul explicite des facteurs significatifs des hétérogénéités du sol par une paramétrisation. Celle-ci va intégrer, à l'échelle de la maille de la grille de discrétisation du calcul (quelques dizaines à quelques centaines de mètres) les effets de ces hétérogénéités sur l'écoulement. On parlera alors de «rugosités complexes» qui dans le cas de la canopée urbaine vont dépendre des caractéristiques locales du site, de la hauteur des bâtiments (moyennes locales et fluctuations autour de ces moyennes), des rapports de ces hauteurs aux largeurs des espaces vides, des rapports des volumes aux surfaces des espaces considérés; toutes mesures qui touchent de près les notions de densité surfacique ou volumique de construction.

Le lancement de l'appel d'offre du PIR-Villes sur les densités a permis de montrer l'intérêt d'une transposition de la notion de densité à celle de rugosité et d'une adaptation, pour la définition des rugosités, de techniques généralement mises en œuvre pour évaluer les densités. Se sont trouvées ainsi associés : les aérodynamiciens de l'Equipe Dynamique de l'Atmosphère Habitée du Laboratoire de Mécanique des Fluides de l'Ecole Centrale de Nantes (URA CNRS 1217), les informaticiens de l'Ecole Centrale de Nantes affiliés à l'Institut de Recherche en Informatique de Nantes et les architectes du Centre de Recherche Méthodologique d'Architecture de l'Ecole d'Architecture de Nantes (URA CNRS 1581). Sans trop alourdir le propos, il faut signaler incidemment que cette opération a marqué le lancement des activités de recherche communes du Pôle «Physique et Images de la Ville». Ce dernier regroupe douze équipes et organismes de recherche de Nantes et du Mans, sous la forme d'une Fédération de Recherche du CNRS.

Les compétences assez contrastées des protagonistes de l'opération se sont avérées de fait très complémentaires, en regard de l'objectif de production semi-automatique des cartes de rugosité, formulé au sein du GDR «subméso». De telles cartes devaient être susceptibles de fournir la paramétrisation convenable des modèles de

\footnotetext{
1. Les codes les plus répandus sont caractérisés par la résolution des équations de Navier-Stokes en 3 dimensions et par l'emploi d'un modèle de turbulence de type "K-Epsilon». D'autres modélisations de la turbulence sont cependant étudiées et préconisées.
}

2. Groleau D., Marenne C., Gadilhe A., "Climatic simulation tools : an application for a building project in an urban space», Communication à 3rd European Conference on Architecture, Solar Energy in Architecture and Urban Planning, Florence, 17-21 may 1993, Bedford. H.S., Stephens \& Associates (1993).

3. Mestayer P.G., Anquetin S., "Climatology of cities" in Diffusion and transport of Pollutants in Atmospheric meso scale How fields, Editors Gyr A., Rysf.S., ErCOftaC Series, Dordrecht, Boston, London, Kluwer Academic Publishers.

4. Groupement de Recherche Atmosphère Urbaine Sub-mesoéchelles. 
simulation numérique des écoulements aérodynamiques en milieu urbain.

Le découpage des tâches imparties à chacune des disciplines s'est retrouvé dans les phases de constitution de cette cartographie de la rugosité. En un premier temps, l'extraction par des moyens informatiques des éléments sur la morphologie d'un territoire urbain, sur la distribution des édifices en regard des espaces libres, des espaces plantés, de la voirie, de l'hydrographie, du relief, impose le choix d'une base de données. Celle-ci doit à la fois livrer les informations directement souhaitées et permettre d'en extraire d'autres, susceptibles d'être recomposées pour construire des indicateurs plus complexes, du type de la densité ou de la rugosité.

\section{Les sources d'information}

L'exploration de l'offre présente en matière de bases de données a été réalisée par les architectes du CERMA. Ces derniers, dans le cadre de travaux sur les instruments de simulation solaire, énergétique et aérodynamique, sont souvent confrontés à la nécessité d'une modélisation numérique des tissus urbains. Tantôt ils construisent leurs propres outils, tantôt ils sont amenés à mettre en œuvre des modeleurs standards, ou à extraire les descriptions morphologiques des fichiers numériques photogéométriques du type des fichiers au format TRAPU réalisés et diffusés par l'IGN Au demeurant, l'expérience du laboratoire en la matière portait davantage sur la restitution de zones de l'importance du fragment urbain que de la totalité d'une aire urbaine. Il est rare, en effet, de posséder pour de tels ensembles des modélisations complètes réalisées à l'aide des outils évoqués (à l'exception près des modćlisations en 3D simplifiées des principales villes françaises, réalisées par l'IGN pour France-Télé- com). Il a fallu, en conséquence, analyser l'offre concernant les bases de données numériques à plus grande échelle et c'est cette investigation qui a représenté la première tâche, dans le registre du recueil des données morphologiques.

Outre ce recueil relatif aux constructions, à l'altimétrie du site, à l'hydrographie ou aux réseaux viaires, il pouvait être intéressant d'obtenir une information a priori sur le type d'occupation et d'usage du sol ; étant admis que cette caractérisation donnait une première approximation de la densité et de la rugosité pour une aire urbaine ou périurbaine donnée.

Différentes bases de données peuvent être sollicitées pour obtenir ce genre d'informations. Dans le cas présent, les systèmes répertoriés ci-après ont été examinés :

- Base B.D. TOPO de l'IGN.

- Base B.D. CARTo de l'IGN.

- Programme Corine-LAND COVER des Communautés Européennes

- Images SPOT de Spot-Image.

En complément, des couvertures plus réduites ont fait l'objet également d'une analyse, afin de comparer les nomenclatures d'occupation du sol utilisées. Il s'agissait des opérations menées par l'IAURP puis par l'IAURIF sur le mode d'occupation des sols de l'agglomération parisienne (MOS) ainsi que l'expérience d'Inventaire Permanent du Littoral (IPLI) qui avait été conduite en 1975 et 1982, et qui intéressait une bande côtière de quelques kilomètres de part et d'autre du rivage. L'opération de la Direction de la Qualité de Vie, des Ressources Humaines et des Moyens du Ministère de l'Environnement sur l'analyse des biosystèmes urbains des agglomérations de plus de 100000 habitants, utilise quant à elle une nomenclature conforme au programme CORINELAND COVER 5 .

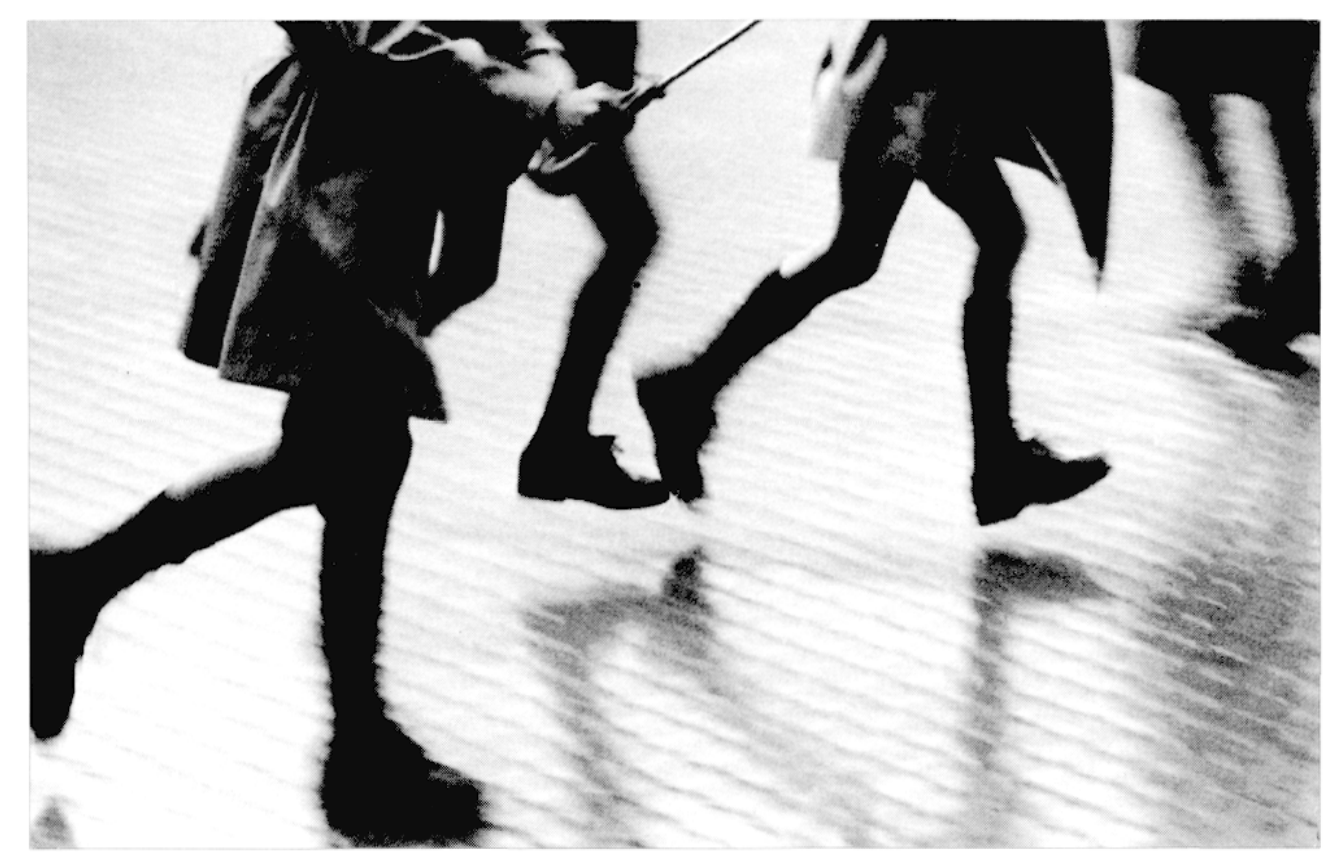


De ce balayage assez général des sources disponibles et des expériences réalisées, deux conclusions ont été tirées pour l'avancement du projet. La première concernait le choix de la base des données permettant d'extraire les informations précises sur la morphologie des constructions et la disposition des lieux urbains considérés. C'est la base B.D. ToPo de l'I.G.N. qui paraissait sur le papier correspondre le mieux aux demandes d'information nécessaire à la réalisation des curtes de rugosité. Un essai d'extraction des données à partir d'un échantillon de cette base a donc été entrepris par les informaticiens de I'I.R.I.N. Ces derniers ont vite constaté que la sélection, dans les fichiers de BD TOPO, des seules données concernant les volumes bâtis utilisant la norme EDIGEO, n'était de densité des zones, grâce notamment, à la distinction entre tissu continu et tissu discontinu. Cette nomenclature, moyennant quelques aménagements, a finalement été retenue pour la suite de l'étude ; demeurent cependant les questions de disponibilité de la couverture correspondante, en fonction de l'avancement du programme CORINE.

\section{Les échelles de rugosité}

Les aérodynamiciens, troisièmes protagonistes de l'opération et partenaires majeurs de celle-ci, dans la mesure où ils en étaient les commanditaires et utilisateurs principaux, sont intervenus dès le démarrage du projet, pour

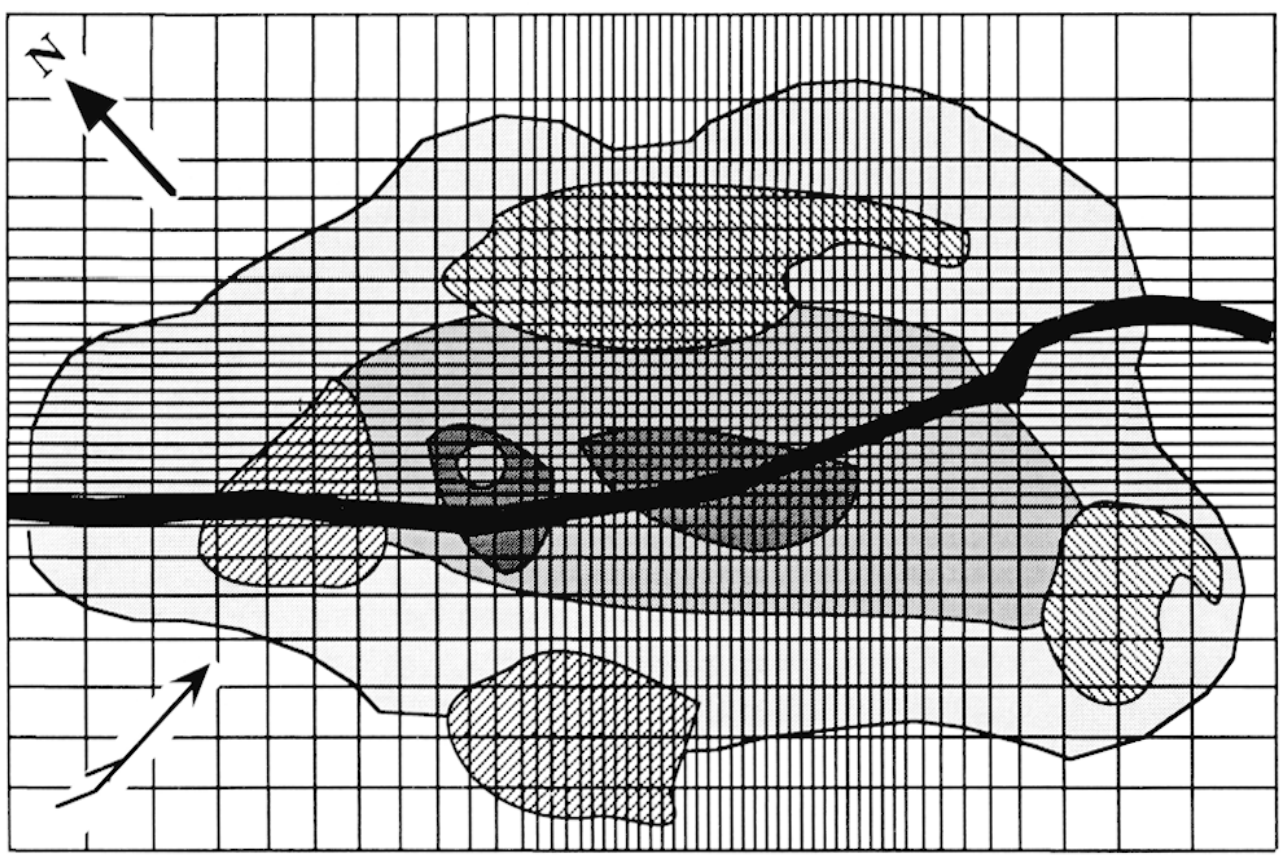

Fig. 3. Schéma montrantle principe du maillage etle raffinement de la grille dans la zone centrale. pas une procédure légère. Parallèlement, une tentative de constitution automatique de cartes de rugosité à partir d'un fichier TRAPU, a été conduite pour Strasbourg (fig. 2).

L'autre enseignement de cette étude préliminaire concernait le choix d'une nomenclature permettant d'effectuer une première classification des types d'occupation des sols. Deux nomenclatures paraissaient mieux adaptées pour répondre au problème posé : celle du MOS de la Région Ile-de-France et celle de CORINELAND COVER. La procédure retenue pour le MOS permet de constituer des légendes distinctes et ne fige pas les passages d'un niveau à l'autre pour toutes les classes. En revanche, elle présente à l'évidence des lacunes, pour ce qui intéresse les zones littorales ou les zones au relief accidenté. La nomenclature CORINE, quant à elle, est totalement déterminée par la notion d'écozone. Son développement limité à trois niveaux permet de subdiviser les classes du troisième niveau pour obtenir une cartographie plus fine. Elle s'avère, en outre, plus apte à rendre compte dès le troisième niveau de l'homogénéité faire partager à l'ensemble des équipes quelques notions claires sur les finalités de l'opération, sur les grandeurs et les procédures physiques qu'elle mettait en œuvre, enfin sur les modalités d'utilisation envisagées pour les descripteurs de la rugosité.

Le fait de laisser un non-spécialiste traduire ce qu'il a retenu des enseignements de mécanique des fluides qui ont occupé les premiers temps de la collaboration, dispensera des formulations algorithmiques qui de toute façon ne sont pas usuelles dans les colonnes des Annales de la Recherche Urbaine.

Dans ces dernières, cependant, deux prédécesseurs avaient alerté les lecteurs sur les arcanes de l'aérodynamique urbaine ${ }^{6}$. Si les éléments sur la couche limite

5. Belchun $B$., «Les sources numériques d'information géographique urbaine", document interne CERMA (mars 94).

6. Sacré C., Gandemer J., "Caractérisation microclimatique des espaces extérieurs du point de vue du vent et de la température", Annales de la Recherche Urbaine n ${ }^{\circ} 61$, mars 1994. 
atmosphérique et la turbulence qui la caractérise dans une mesure d'autant plus forte que la hauteur des constructions s'accroît, n'ont pas besoin d'être repris, le propos des spécialistes du CSTB était plus orienté vers l'usage des outils de simulation analogique en souffleries climatiques, que vers les procédures numériques. Il n'est, au demeurant, pas question d'amorcer ici une vaine querelle sur les deux modes d'investigation. Il est patent qu'ils répondent à des demandes d'ordre différent, s'inscrivant dans des procédures d'application opérationnelle immédiate pour les uns et de recherche à plus long terme pour les autres.

Quoi qu'il en soit, si l'on est confronté au problème de reconstitution des mouvements aérodynamiques en milieu urbain, on n'échappe pas à la nécessité de caractériser l'écoulement par une grandeur correspondant à la rugosité. Dans le cas d'un écoulement au contact d'une paroi homogène, cette grandeur peut-être reliée empiriquement à la hauteur moyenne des aspérités de la paroi. Mais le rapport entre cette mesure de rugosité et la hauteur moyenne de ces aspérités n'est pas connu dans le cas où la surface n'est pas homogène. Inutile d'insister sur le fait que cette inhomogéneité caractérise fortement la nature des zones urbaines. A leur propos, pour donner une mesure au paramètre de rugosité, deux approches sont possibles.

L'approche géométrique rejoint la méthode utilisée pour le cas homogène. Elle consiste à tenter de relier empiriquement l'indicateur de rugosité à une échelle géométrique de forme qui est équivalente à la hauteur moyenne et qui est construite à partir de paramètres jugés représentatifs de la morphologie locale du tissu urbain considéré. A ce stade, des hypothèses doivent être faites sur la pertinence des paramètres en question tandis que des tests permettront ensuite de les valider. Dans le cadre du projet, on a examiné les indicateurs suivants :

- la hauteur moyenne des bâtiments ;

- la hauteur surfacique ;

- l'écart-type des hauteurs ;

- la hauteur frontale moyenne ;

- la densité surfacique ;

- le rapport de forme frontal ;

- le rapport de forme horizontal ;

En regard de cette procédure nécessitant des calculs précis pour chaque simulation, on peut souhaiter identifier des catégories de tissus urbains possédant des caractéristiques de rugosité connues et relativement homogènes. Cette approche typologique implique la détermination, pour chaque catégorie de tissu retenu, de l'indicateur de rugosité. Des travaux de recherche sont menés en ce sens par l'équipe Dynamique de l'Atmosphère Habitée, pour déterminer des fonctions empiriques de la densité, de la hauteur moyenne des bâtiments et de leur hauteur frontale moyenne, afin de caractériser la rugosité pour un type de tissu donné.

\section{Echelle de résolution et discrétisation spatiale}

L'échelle retenue pour les études d'aérodynamique urbaine se situe entre celle d'un quartier et celle d'une agglomération, soit un intervalle de distances allant de trois à une quarantaine de kilomètres. L'échelle d'analyse dépend de celle des mailles du calcul. Elle résulte des limitations actuelles des supercalculateurs? ${ }^{7}$. Selon la résolution du maillage et la complexité des modèles dynamiques, le maillage au sol est limité dans une fourchette de $10^{4}$ à $10^{5}$ nœuds, ce qui conduit à une résolution pour la grille horizontale comprise entre $10 \mathrm{~m} \times 10 \mathrm{~m}$ et $300 \mathrm{~m} \times 300 \mathrm{~m}$.

Les codes de calcul sont pour leur quasi-totalité développés à partir de méthodes aux différences finies ou aux volumes finis. C'est le cas du code ARPS, destiné à devenir l'outil commun des équipes françaises pour l'étude des écoulements «sub-méso». Le maillage horizontal du calcul est quadrangulaire (cartésien ou curvilinéaire) et généralement non homogène. La figure stylisée (fig. 3) montre comment ce maillage permct un raffinement de grille dans la zone du centre ville. Les limitations de ce type de maillage pour des cas complexes sont évidentes. En effet, certains éléments ont une importance particulière pour la détermination des champs de vitesse et de turbulence de l'écoulement (zone au relief marqué, constructions de grandes hauteurs). Ils ne se trouvent pas toujours au centre de la zone d'étude et vont induire une complexification du resserrement du maillage. Enfin, pour tenir compte des irrégularités d'implantation liées, par exemple, à un cours d'eau, le maillage sera le plus souvent curvilinéaire et sera généré par blocs à l'aide d'un opérateur elliptique, muni de fonctions de resserrement local. Il n'en restera pas moins quadrangulaire et les variables dépendantes scalaires telles que la rugosité, seront définies au centre des mailles et représenteront la valeur intégrale sur le domaine limité par la maille.

\section{La procédure en cours de développement}

A ce stade de l'exposé, il faut espérer que l'on a pu faire saisir la nature des éléments dont on disposait et la nécessité de définir, à partir de ces derniers, une valeur caractéristique de l'échelle de rugosité. Ceci pour toute maille de la grille de calcul de l'écoulement. Une des techniques envisagée consiste à superposer une carte de rugosité à la grille horizontale (projection au sol du maillage). C'est la réalisation de cette carte à partir des bases de données urbaines qui représente l'enjeu majeur du projet. Elle ne

7. Ces calculs sont réalisés actuellement par l'équipe Dynamique de l'Atmosphère Habitée sur le CRAY C98 du Centre de Calcul du CNRS et sur le VAX 9420 du Centre de Ressources Informatiques de l'Ecole Centrale de Nantes. Ce calculateur est également celui utilisé par le CERMA pour réaliser les simu. lations présentées dans cet article. 
marque pas pour autant son achèvement, étant entendu que des phases de validation prenant la forme de calculs comparatifs d'écoulements, doivent compléter le programme de recherche.

Le passage de la base de données urbaines et géographiques à la carte de rugosité n'a pas le caractère d'une transcription mécanique où se poseraient seulement quelques problèmes de délimitation de zones homogènes. Un certain degré d'inter-activité el d'ajustement des paramétrages est requis pour l'investigation aérodynamique. Cette perspective vient modifier quelque peu l'image d'un automatisme rigide, qu'une présentation par trop schématique de la procédure risquerait de donner.

A titre indicatif on pourrait envisager qu'elle se déroule suivant un enchaînement organisé comme suit :

- lecture du domaine géographique couvert par la banque de données retenue ;

- délimitation du domaine d'étude par l'utilisateur aérodynamicien ;

- calcul et affichage pour ce domaine de la typologie des zones extraite de la même base de données ou reportée à partir d'une autre base ;

- définition des blocs (ajustés sur les contours des sousdomaines précédents) et de la densité du maillage de calcul de simulation, génération de ce maillage ;

- affichage de la topographie ;

- définition des fonctions de resserrement du maillage, création de nouveaux blocs, raffinage de la résolution de certains blocs; génération de maillage aérodynamique raffiné ;

- calcul des paramètres morphologiques (densité, hauteur moyenne, hauteur frontale moyenne...) sur le maillage aérodynamique ;

- sélection des paramètres permettant de caractériser la rugosité, affichage de la carte correspondante à la résolution initiale (celle des contours de la base de données) et à la résolution du maillage, comparaison ;

- fin de la procédure ou retour en un point quelconque antérieur8.

Il faut en conséquence, augurer qu'au terme de cette recherche, le nouvel instrument réalisé permettra tant l'affichage des cartes isovaleurs d'un paramètre de rugosité calculé ou la délimitation des zones typologiques, que la modification interactive du degré de précision de la restitution. Il devra en outre fournir la possibilité d'isoler manuellement certaines zones, de réaliser un traitement statistique différent d'une zone à l'autre, enfin de donner pour les paramètres de rugosité sélectionnés, un fichier de ces paramètres à la résolution du maillage aérodynamique.

\section{Quelques enseignements et perspectives}

Ces dernières considérations ont un caractère votif dans la mesure où les fonctionnalités évoquées ne sont pas, loin s'en faut, toutes opérationnelles. Le programme de recherche se trouvant à mi-parcours, une telle présentation «en cours d'exécution» pèche bien sûr par l'absence de résultats tangibles et se limite à la description d'un état d'avancement. On peut assortir ce dernier d'éléments d'un ordre tout différent sur les conditions de mise en œuvre du projet. Le travail commun entre spécialistes d'horizons très divers a certes les mérites de l'ouverture et de l'enrichissement, il connaît aussi une face plus sombre.

Cet autre éclairage peut être donné à propos des modalités de collaboration qui se sont nouées, dans le cas présent, entre spécialistes d'obédiences scientifiques fort variées. Même si certains avaient quelques propensions à s'évader de leurs strictes clôtures disciplinaires, le travail en commun n'a pas toujours été exempt de difficultés et de malentendus. Dans ce jeu à trois, il s'est avéré souvent difficile de faire fonctionner le groupe à l'unisson. Suivant des schémas bien connus, des doublets de connivence et de coopération se sont constitués tour à tour. La dynamique du projet a porté jusque là davantage sur ces synergies binaires; elles voyaient à un moment précis architectes et aérodynamiciens se retrouver en parfait accord pour une phase donnée, puis architectes et informaticiens coopérer pour une autre séquence et ainsi de suite. En dépit de ce constat, la connaissance mutuelle des problématiques, des techniques et des modes d'approche, mais aussi des obstacles rencontrés par les uns et les autres, a indéniablement progressé.

Cette amélioration n'est pourtant pas un objectif suffisant. Pour s'inscrire dans une véritable logique interdisciplinaire, il conviendrait de construire des entités scientifiques nouvelles communes à plusieurs champs de disciplines. On souhaiterait bien sûr que le rejeton quelque peu bizarre de la densité que nous avons retenu sous le vocable de «rugosité» puisse s'exporter au-delà du domaine de l'aérodynamique. La perspective n'est peutêtre pas aussi artificielle qu'il y paraitrait en première approche.

$\mathrm{Ne}$ pourrait-on pas dépasser le point de vue d'Eole et montrer qu'au-delà de la simulation des écoulements du vent, la démarche entreprise et les indicateurs mis au jour pourraient fournir des informations extensives sur la perméabilité du bâti à d'autres phénomènes constitutifs des ambiances urbaines? On songe en particulier aux ambiances lumineuses, aux propagations sonores, aux caractérisations thermiques et radiatives. Il est bien clair que de telles rêveries sont encore loin de toute problématisation scientifique, s'il est admis, en revanche, qu'elles peuvent constituer le germe de ces dernières.

Jean-Pierre Peneau

8. Mestayer P.G., Sini J.-F., "Aérodynamique et rugosité", document interne D.A.H (mars 94). 